\title{
Back Squat Potentiates Both Vertical and Horizontal Jump Performance in Collegiate Ice Hockey Players
}

\author{
Cale Bechtel, Joshua A. Cotter, Evan E. Schick* \\ Physiology of Exercise and Sport (PEXS) Laboratory, Department of Kinesiology, Long Beach State University, 1250 Bellflower Blvd., \\ Long Beach, CA, 90840-4901
}

Corresponding Author: Evan E. Schick, E-mail: evan.shick@csulb.edu

\section{ARTICLE INFO}

Article history

Received: November 12, 2017

Accepted: January 22, 2018

Published: January 31, 2018

Volume: 6 Issue: 1

Conflicts of interest: None

Funding: None

\begin{abstract}
Background: Back squats (BSQ) have been shown to transiently improve performance in explosive vertical movements such as the vertical jump (VJ). Still, understanding of this phenomenon, termed post-activation potentiation (PAP), remains nebulous as it relates to explosive horizontal movements. Objective: Therefore, the purpose of the present investigation was to assess whether heavy BSQ can potentiate both VJ and horizontal jump (HJ) performance. Method: Nine male ice hockey players from the Long Beach State ice hockey team performed five testing sessions separated by 96 -hours. The first testing session consisted of a one repetition maximum (1-RM) BSQ to determine subsequent testing loads. The four subsequent testing sessions, which were randomized for order, consisted of five repetitions of BSQ at 87\% 1-RM followed by horizontal jump (BSQ-HJ), five repetitions of BSQ at 87\% 1-RM followed by vertical jump (BSQ-VJ), horizontal jump only (CT-HJ) and vertical jump only (CT-VJ). During the potentiated conditions, rest intervals were set at five minutes between the BSQ and either VJ or HJ. Alpha-level was set a priori at 0.05. Results: The results indicate that both vertical $(\mathrm{p}=0.017)$ and horizontal $(\mathrm{p}=0.003)$ jump were significantly increased $(\mathrm{VJ}=+5.51 \mathrm{~cm}$, $\mathrm{HJ}=+11.55 \mathrm{~cm}$ ) following a BSQ. Conclusion: These findings suggest that BSQ may improve both vertical and horizontal jump performance in athletes who participate in sports emphasizing horizontal power, such as ice hockey.
\end{abstract}

Keywords: Plyometric Exercise, Muscle Strength, Resistance Training, Back Squat, Acute Exercise

\section{INTRODUCTION}

Heavy-load lifting has been shown to transiently improve muscular power (Hodgson, Docherty and Robbins, 2005, Tillin and Bishop, 2009). This phenomenon, termed post activation potentiation (PAP), is maximized by performing an explosive movement task subsequent to a heavily loaded lift that shares a similar movement pattern (Golas, Maszczyk, Zajac, Mikolajec and Stastny, 2016). PAP is influenced by a combination of factors such as volume and intensity, intraset and intertask rest intervals, participant characteristics and the type of exercise performed (Hodgson, Docherty and Robbins, 2005, Seitz and Haff, 2016, Seitz, Mina and Haff, 2016, Tillin and Bishop, 2009, Tobin and Delahunt, 2014). Used effectively in a training program, PAP may enhance sensitivity to power training such as plyometrics (Docherty and Hodgson, 2007, Tillin and Bishop, 2009). As peak performance in most sports is a function of maximizing muscular power, PAP training may be an important asset to resistance training programs (Docherty and Hodgson, 2007).

Back squats (BSQ), which are ubiquitously used in exercise training, have consistently been shown to potentiate vertical jump (VJ) performance across various populations (Docherty and Hodgson, 2007, Evetovich, Conley and McCawley, 2015, Seitz and Haff, 2016, Seitz, Mina and Haff, 2016, Tillin and Bishop, 2009, Tobin and Delahunt, 2014). $\mathrm{VJ}$ height is a measure of lower body power that is commonly used as an indicator of athletic potential since explosive leg power correlates to performance in many sports (Burkett, Phillips and Ziuraitis, 2005, Gourgoulis, Aggeloussis, Kasimatis, Mavromatis and Garas, 2003). Robust VJ potentiation occurs following BSQ performed with loads of up to $87 \%$ of the one repetition maximum (1-RM) and four to eight minutes of post-squat rest (Hodgson, Docherty and Robbins, 2005, Kilduff, Owen, Bevan, Bennett, Kingsley and Cunningham, 2008, Nibali, Chapman, Robergs and Drinkwater, 2015, Seitz and Haff, 2016, Tillin and Bishop, 2009). The apparent efficacy of BSQ-VJ potentiation may be due to shared activation patterns in lower body musculature, including: quadriceps, hamstrings, gastrocnemius, gluteus maximus, gluteus medius, plantar flexors, dorsiflexors, abdominals and spinal erectors (Delavier, 2010, Docherty and Hodgson, 2007, Gullett, Tillman, Gutierrez and Chow, 2009, 
Seitz and Haff, 2016, Tillin and Bishop, 2009). Although VJ predominates PAP research, it is imperative to explore alternative movement patterns that may be potentiated by BSQ as certain sports do not emphasize vertical movement patterns.

One such sport is ice hockey, which emphasizes horizontal movement patterns instead of vertical. In fact, the musculoskeletal fitness assessment section of the National Ice Hockey League (NHL) combine utilizes standing horizontal jump performance as their primary means of assessing muscular power (Glendhill, 2007). High scores on this assessment indicate a player's ability to skate powerfully, shoot hard, and push opponents out of the way (Glendhill, 2007). Yet, a review of current PAP research reveals a dearth of literature surrounding the sport of ice hockey as well as the nature of horizontal jump potentiation; to date, just one study using rugby players has reported that loaded BSQ can potentiate HJ performance (Seitz, Mina and Haff, 2016). Furthermore, insufficient data exists in support of whether the aforementioned BSQ-VJ potentiation extends to athletes specializing in sports which stress horizontal movement. Therefore, the present study examined collegiate ice hockey players to pursue the following aims: 1) to clarify whether BSQ-VJ potentiation extends to athletes competing in horizontal movement-dominant sports, 2) to assess whether HJ is also sensitive to BSQ-induced potentiation, and 3 ) to compare levels of $\mathrm{VJ}$ and $\mathrm{HJ}$ potentiation. The primary hypothesis follows that heavy BSQ will potentiate both VJ and HJ performance.

\section{MATERIALS AND METHODS}

\section{Experimental Approach}

Participants performed all five testing sessions. The first testing session consisted of a 1-RM BSQ. The four remaining testing sessions, performed in random order, consisted of: 1) back squat followed by horizontal jump (BSQ-HJ), 2) back squat followed by vertical jump (BSQ-VJ), 3) horizontal jump only (CT-HJ) and 4) vertical jump only (CT-VJ). Participants completed five BSQ repetitions at $87 \%$ of their $1-\mathrm{RM}$ during the BSQ-HJ and BSQ-VJ testing sessions. Table 1 summarizes the study design.

\section{Participants}

Nine male ice hockey players $(22 \pm 0.47$ years $)$ from the Long Beach State University club ice hockey team voluntarily participated for this study (Table 2). Participants were college level athletes with experience in ice hockey (11 \pm 1.47 years) and resistance training ( $4 \pm 0.94$ years). The Institutional Review Board of Long Beach State University approved the research protocol. Participants signed a Physical Activity Readiness Questionnaire (PAR-Q) and informed consent before participation. Inclusion criteria consisted of: 1) active member of LBSU ice hockey team, 2) free from unresolved orthopedic injuries for the preceding 12 months, 3) free from diagnosed cardiopulmonary or metabolic disease, 4) two or more years of resistance training experience, and 5) five or more years of ice hockey experience.

\section{Instrumentation and Tools}

For the BSQ, a 45lb standard Olympic barbell and bumper plates $\left(45,35,25,15,10\right.$ lbs.) were utilized (Rogue ${ }^{\circledR}$ Columbus, OH). Participants performed the BSQ inside a squat rack (Rogue ${ }^{\circledR}$ Columbus, OH). A Vertec ${ }^{\circledR}$ (Sports Imports Inc., $\mathrm{OH}$ ) jumping apparatus was used to measure vertical jump (VJ) height to the nearest $(\mathrm{cm})$. The horizontal jump (HJ) performance test was assessed on the floor using a cloth tape measure with increments to the nearest $(\mathrm{cm})$ (Jackson, 2017).

\section{Procedures}

Testing encompassed five separate sessions, each separated by 96-hours, during which participants refrained from resistance exercise. Past reports confirm robust VJ potentiation following BSQ performed with loads of to 87\% 1-RM and four to eight minute post-squat rest periods (Hodgson, Docherty and Robbins, 2005, Kilduff, Owen, Bevan, Bennett, Kingsley and Cunningham, 2008, Seitz and Haff, 2016, Seitz, Mina and Haff, 2016, Tillin and Bishop, 2009). Thus, we chose a BSQ intensity level of 87\% 1-RM as well as five minute post-squat rest periods for the BSQ-VJ and BSQ$\mathrm{HJ}$ conditions. The first testing session consisted of a 1-RM BSQ in order to calculate the load corresponding to $87 \%$ of the 1-RM load. All participants successfully performed five repetitions of BSQ at $87 \% 1-\mathrm{RM}$ during the BSQ-VJ and BSQ-HJ testing sessions. The 1-RM BSQ protocol was adopted from the National Strength and Conditioning Association (Baechle, 2008). Briefly, participants first performed five to ten repetitions at a light resistance $(<30 \%$ projected $1-\mathrm{RM})$ as part of a squat-specific warm-up. Following a one minute rest period, three to five more repetitions were performed with an additional 30-40lbs. Participants then rested for two minutes before performing two to three repetitions with an additional 30-40lbs. All subsequent sets included two minute rest periods and only single repetitions with incremental increases between 10-40 lbs. until the 1-RM was completed. Load was incrementally added to achieve the 1-RM within 3-5 sets. A general warm up protocol for all five testing sessions (1-RM, BSQ-HJ, BSQ-VJ, CT-HJ, CT-VJ) began with a three to five minute warm up on a stationary bike at a self-selected resistance. A choice was given after the three minute mark whether they wanted to continue until five minutes or began their mandatory athletic drills and ballistic stretches. Next a series of athletic drills (internal/external hip rotations, lateral shuffles, high knees, butt kicks) were performed. Athletic drills were performed twice over the length of the laboratory. Two ballistic stretching exercises (frontal and lateral leg swings) were utilized after the athletic drills prior to beginning the testing session (Delavier, 2010, Ojeda, Rios, Barrilao and Serrano, 2016, Tobin and Delahunt, 2014). Participants performed five repetitions using $87 \%$ of their 1-RM load for the BSQ-HJ and BSQ-VJ conditions. Participants were encouraged to use a squat stance width and bar placement that was consistent with their typical technique. Depth of the squat was strictly enforced; terminal range was reached once the inguinal 
Table 1. Study Design

\begin{tabular}{lcccccc}
\hline Condition & Sets & Reps & $\begin{array}{c}\text { S.I. (\%) } \\
\text { 1-RM) }\end{array}$ & R.I. (min) & HJ (cm) & VJ (cm) \\
\hline 1-RM & $3-5$ & NSCA Protocol & - & - & - & - \\
BSQ-HJ & 1 & 5 & $87 \%$ & 5 & HJ & - \\
BSQ-VJ & 1 & 5 & $87 \%$ & 5 & - & VJ \\
CT-HJ & 0 & 0 & $0 \%$ & Warm up & HJ \\
CT-VJ & 0 & 0 & $0 \%$ & Warm up & - & VJ \\
\hline
\end{tabular}

1-RM=Back Squat Max Test; BSQ=Back Squat; CT=Control; HJ=Horizontal Jump; VJ=Vertical Jump;S.I. = Squat Intensity; R.I. = Rest Interval

crease and proximal portion of the knee were level (Baechle, 2008, Reardon, Hoffman, Mangine, Wells, Gonzalez, Jajtner, Townsend, McCormack, Stout, Fragala and Fukuda, 2014). Squat depth was visually monitored by two certified strength and conditioning specialists (CSCS), one on each side of the lifter. Following the BSQ, participants rested for 5 minutes before performing either the VJ or HJ (Ray, 2017). The NHL Entry Draft protocol was used to assess HJ (Glendhill, 2007). The participants stood with feet slightly apart with their toes behind the jumping line. Using an arm swing the participants jumped as far as possible, and the average of the three trials was used. Distance was recorded to the nearest $(\mathrm{cm})$ from the jumping line to the heel mark utilizing a cloth tape measure. The VJ was assessed using a Vertec $\AA$ apparatus following the National Strength and Conditioning Association vertical jump protocol (Baechle, 2008) Participants were not required to squat to a certain depth. VJ was recorded to the nearest $1 / 4$ inch from the average of three jumps and later converted to $(\mathrm{cm})$.

\section{Statistical Analysis}

$\mathrm{VJ}$ and $\mathrm{HJ}$ potentiation was assessed by paired samples t-tests (SPSS version 24). Effect sizes were determined by Cohen's d for paired samples t-test (Lakens, 2013), which assessed the magnitude of the change in $\mathrm{VJ}$ and $\mathrm{HJ}$ performance following BSQ. These changes were considered trivial $<0.20$; small, $0.20-0.50$; medium, $0.5-0.8$; large, $0.8-1.30$; or very large, $>1.30$ (Hopkins, 2010). Shapiro-Wilk test confirmed normality for all conditions ( $\mathrm{Sig}>0.05)$. Alpha-level was set a priori at 0.05 .

\section{RESULTS}

The characteristics of participants are illustrated in table 2 . Our primary objective was to examine the effect of BSQ on $\mathrm{VJ}$ and $\mathrm{HJ}$ performance in athletes participating in horizontal movement-dominant sports. To this end, we subjected nine collegiate ice hockey players to four randomized testing sessions (CT-VJ, BSQ-VJ, CT-HJ, BSQ-HJ). VJ performance was significantly increased $(+5.51 \mathrm{~cm})$ following the BSQ $(\mathrm{p}=0.017)$. HJ performance was also significantly increased $(+11.55 \mathrm{~cm})$ following the BSQ $(\mathrm{p}=0.003)$. Additionally, Cohen's d revealed a large effect size for both VJ (1.00) and $\mathrm{HJ}$ (1.38). Table 3 summarizes the horizontal and vertical jump distances in both testing conditions.
Table 2. Subject Descriptive Data

\begin{tabular}{lc}
\hline Variables & Mean $\pm \boldsymbol{S D}$ \\
\hline Height $(\mathrm{cm})$ & $188.62 \pm 2.73$ \\
Weight $(\mathrm{kg})$ & $87.94 \pm 20.49$ \\
RT $(\mathrm{yrs})$ & $4.02 \pm 2.82$ \\
HE (yrs) & $11.01 \pm 4.41$ \\
Age (yrs) & $22.04 \pm 1.41$ \\
1-RM & $86.22 \pm 35.19$ \\
\hline
\end{tabular}

$\mathrm{SE}=$ Standard Deviation RT=Resistance Training Experience

$\mathrm{HE}=$ Ice Hockey Experience

Table 3. Horizontal and Vertical Jump

Distance (mean \pm standard error)

\begin{tabular}{llcc}
\hline Condition & $\boldsymbol{n}$ & Jump Distance $(\mathbf{c m})$ & Effect Size \\
\hline CT-VJ & 9 & $54.45 \pm 3.34$ & 1.00 \\
BSQ-VJ & 9 & $59.96 \pm 3.20 \dagger$ & \\
CT-HJ & 9 & $199.35 \pm 8.51$ & 1.38 \\
BSQ-HJ & 9 & $210.92 \pm 7.98^{*}$ & \\
\hline
\end{tabular}

CT-HJ=Horizontal Jump - Control; BSQ-HJ=Back

Squat - Horizontal Jump;CT-VJ=Vertical

Jump - Control; BSQ-VJ=Back Squat - Vertical Jump; ${ }^{*} \mathrm{P}<0.05$

versus $\mathrm{CT}-\mathrm{HJ} ; \uparrow \mathrm{P}<0.05$ versus $\mathrm{CT}-\mathrm{VJ}$

\section{DISCUSSION}

The present study examined whether loaded BSQ could potentiate VJ and $\mathrm{HJ}$ performance in collegiate ice hockey players. Herein, we demonstrate that performing five repetitions of BSQ at 87\% 1-RM significantly potentiates both $\mathrm{VJ}$ and HJ performance in collegiate ice hockey players. Furthermore, effect size (ES) calculation revealed a large (ES $>0.8$ ) magnitude of improvement for each jump following BSQ. The current findings concerning VJ performance have been thoroughly corroborated by past studies which have established that potentiation may occur four to eight minutes following near maximal intensity (>80\% $1-\mathrm{RM})$ dynamic movements or isometric contractions (Evetovich, Conley and McCawley, 2015, Nibali, Chapman, Robergs and Drinkwater, 2015, Seitz, Mina and Haff, 2016, Tillin and Bishop, 2009, Tobin and Delahunt, 2014). Additionally, our data are in-line with past PAP research which has shown significant VJ potentiation following a BSQ at 87\% 1-RM (Kilduff, Owen, Bevan, Bennett, Kingsley and Cunningham, 2008, Reardon, Hoffman, Mangine, Wells, Gonzalez, 
Jajtner, Townsend, McCormack, Stout, Fragala and Fukuda, 2014). In the present study, BSQ significantly potentiated VJ $(\mathrm{p}=0.017)$, and the magnitude of this effect was large $(\mathrm{ES}=$ 1.00). These findings are consistent with several other PAP studies using similar procedures (Evetovich, Conley and McCawley, 2015, Nibali, Chapman, Robergs and Drinkwater, 2015, Seitz, Mina and Haff, 2016). Still, to our knowledge, this study is the first to investigate and produce data indicating that BSQ can potentiate VJ performance in collegiate ice hockey players. This suggests that vertical movement patterns can be acutely potentiated in athletes who primarily perform in a horizontal direction. Future research should explore whether vertical potentiation in training can improve an ice hockey player's ability to skate powerfully, shoot hard, and push opponents out of the way.

HJ performance is used as an assessment for lower body power in sports which predominately move in a horizontal direction, such as ice hockey (Glendhill, 2007). This suggests that, 1) improving horizontal power may be advantageous to ice hockey performance, and 2) improving HJ performance may increase the likelihood of being drafted by an ice hockey team. Nevertheless, an overall paucity of data exists regarding $\mathrm{HJ}$ potentiation. The current study is the first to explore HJ potentiation in ice hockey players. Our data show that HJ was significantly potentiated by the BSQ $(p=0.003)$ with a mean increase of $(+11.55 \mathrm{~cm})$, and, similar to BSQ-VJ, the magnitude of this effect was large $(E S=1.38)$. These findings are supported by one previous report in which multiple sets of BSQ potentiated HJ in rugby players (Seitz, Mina and Haff, 2016). As noted above, ice hockey movement patterns are primarily in a horizontal direction, thus this finding may suggest that athletes competing predominately in a horizontal direction are sensitive to horizontal movement potentiation. However, future research should explore whether this phenomenon is exclusive to athletes in horizontal movement sports or if athletes who participate in non-horizontal dominant sports experience similar horizontal movement potentiation. A noteworthy limitation of the current study is that of relative BSQ strength. Relative BSQ strength is a key variable in facilitating movement potentiation (Seitz and Haff, 2016, Tillin and Bishop, 2009). Previous reports suggest that PAP training complexes are maximized in male athletes who can BSQ 1.75x their bodyweight (Seitz and Haff, 2016). In the current study, participants back squatted an average of $0.97 \mathrm{x}$ their bodyweight. Though the implication of this limitation is unclear, it is possible that greater BSQ performance could have increased effect sizes in both VJ and HJ. Thus, future endeavors should examine the interaction of BSQ strength with both HJ and VJ as well as how this potential interaction compares between the two types of jumps.

\section{CONCLUSION}

In conclusion, findings from the present study demonstrate that five repetitions of BSQ, performed at 87\% 1-RM, can potentiate VJ and HJ performance. These findings suggest that strength and conditioning practitioners may benefit from incorporating potentiation complexes in order to improve horizontal power in athletes who must excel in a horizontal direction. Future research should explore the extent to which varying levels of relative BSQ strength may influence the magnitude of $\mathrm{VJ}$ and $\mathrm{HJ}$ potentiation, and whether horizontal potentiation is expressed by athletes who participate in non-horizontal dominant sports.

\section{REFERENCES}

Baechle TR, Earle, R.W. (2008) Essentials of Strength and Conditioning. Champaign, IL: Human Kinetics.

Burkett LN, Phillips WT, Ziuraitis J. (2005) The best warm-up for the vertical jump in college-age athletic men. Journal Of Strength And Conditioning Research 19(3):673-6.

Delavier F. (2010) Strength Training Anatomy. $3^{\text {rd }}$ ed. Champaign, Illinois: Human Kinetics.

Docherty D, Hodgson MJ. (2007) The application of postactivation potentiation to elite sport. International Journal Of Sports Physiology And Performance 2(4):439-44.

Evetovich TK, Conley DS, McCawley PF. (2015) Postactivation potentiation enhances upper- and lower-body athletic performance in collegiate male and female athletes. Journal Of Strength And Conditioning Research 29(2):336-42.

Glendhill N, \& Jannik, V. (2007) Detailed assessment protocols for NHL entry draft players. Toronto, ONT: York University.

Golas A, Maszczyk A, Zajac A, Mikolajec K, Stastny P. (2016) Optimizing post activation potentiation for explosive activities in competitive sports. Journal Of $\mathrm{Hu}$ man Kinetics 52:95-106.

Gourgoulis V, Aggeloussis N, Kasimatis P, Mavromatis G, Garas A. (2003) Effect of a submaximal half-squats warm-up program on vertical jumping ability. Journal Of Strength And Conditioning Research 17(2):342-4.

Gullett JC, Tillman MD, Gutierrez GM, Chow JW. (2009) A biomechanical comparison of back and front squats in healthy trained individuals. Journal Of Strength And Conditioning Research 23(1):284-92.

Hodgson M, Docherty D, Robbins D. (2005) Post-activation potentiation: underlying physiology and implications for motor performance. Sports Medicine 35(7):585-95.

Hopkins WG. (2010) Linear models and effect magntidues for research, clinical and practical applications. Sportscience 14:49-57.

Jackson KM, Beach T.A. and Andrews D.M. (2017) The effect of an isometric hip muscle strength training protocol on valgus angle during a drop vertical jump in competitive female volleyball players. International Journal of Kinesiology \& Sports Science 5(4):1-9.

Kilduff LP, Owen N, Bevan H, Bennett M, Kingsley MI, Cunningham D. (2008) Influence of recovery time on post-activation potentiation in professional rugby players. Journal Of Sports Sciences 26(8):795-802.

Lakens D. (2013) Calculating and reporting effect sizes to facilitate cumulative science: a practical primer for t-tests and ANOVAs. Frontiers in Psychology 4:863. 
Nibali ML, Chapman DW, Robergs RA, Drinkwater EJ. (2015) Considerations for determining the time course of post-activation potentiation. Applied Physiology, $\mathrm{Nu}$ trition, And Metabolism 40(11):1163-70.

Ojeda AH, Rios LC, Barrilao RG, Serrano PC. (2016) Acute effect of a complex training protocol of back squats on 30-m sprint times of elite male military athletes. Journal of Physical Therapy Science 28(3):752-6.

Ray T, Adams, KJ, DeBeliso, M. (2017) The Relationship Between Core Stability \& Squat Ratio in Resistance-Trained Males. International Journal of Kinesiology \& Sports Science 5(2):7-15.

Reardon D, Hoffman JR, Mangine GT, Wells AJ, GonzalezAM, Jajtner AR, Townsend JR, McCormack WP, Stout JR, Fragala MS, Fukuda DH. (2014) Do changes in muscle architecture affect post-activation potentiation? Journal Of Sports Science \& Medicine 13(3):483-92.
Seitz LB, Haff GG. (2016) Factors Modulating Post-Activation Potentiation of Jump, Sprint, Throw, and Upper-Body Ballistic Performances: A Systematic Review with Meta-Analysis. Sports Medicine 46(2):231-40.

Seitz LB, Mina MA, Haff GG. (2016) Postactivation Potentiation of Horizontal Jump Performance Across Multiple Sets of a Contrast Protocol. Journal Of Strength And Conditioning Research 30(10):2733-40.

Tillin NA, Bishop D. (2009) Factors modulating post-activation potentiation and its effect on performance of subsequent explosive activities. Sports Medicine 39(2):147-66.

Tobin DP, Delahunt E. (2014) The acute effect of a plyometric stimulus on jump performance in professional rugby players. Journal Of Strength And Conditioning Research 28(2):367-72. 